\title{
Increased hepcidin expression in multibacillary leprosy
}

\author{
Vânia Nieto Brito de Souza', Tatiana Salles de Souza Malaspina² ${ }^{2}$ Ana Paula Campanelli², \\ Cássio Ghidella ${ }^{3}$, Somei Ura' ${ }^{1}$, Dirceu Dalpino', Dejair Caitano do Nascimento ${ }^{1}$, Ana Carla Pereira Latini ${ }^{1 /+}$ \\ ${ }^{1}$ Instituto Lauro de Souza Lima, Secretaria de Estado da Saúde, Bauru, SP, Brasil ²Departamento de Ciências Biológicas, \\ Faculdade de Odontologia de Bauru, Universidade de São Paulo, Bauru, SP, Brasil \\ ${ }^{3}$ Secretaria de Saúde do Município de Rondonópolis, Rondonópolis, MT, Brasil
}

Iron is essential for all organisms and its availability can control the growth of microorganisms; therefore, we examined the role of iron metabolism in multibacillary (MB) leprosy, focusing on the involvement of hepcidin. Erythrograms, iron metabolism parameters, pro-inflammatory cytokines and urinary hepcidin levels were evaluated in patients with $M B$ and matched control subjects. Hepcidin expression in MB lesions was evaluated by quantitative polymerase chain reaction. The expression of ferroportin and hepcidin was evaluated by immunofluorescence in paucibacillary and MB lesions. Analysis of hepcidin protein levels in urine and of hepcidin mRNA and protein levels in leprosy lesions and skin biopsies from healthy control subjects showed elevated hepcidin levels in MB patients. Decreases in haematologic parameters and total iron binding capacity were observed in patients with MB leprosy. Moreover, interleukin-1 beta, ferritin, soluble transferrin receptor and soluble transferrin receptor/log ferritin index values were increased in leprosy patients. Hepcidin was elevated in lepromatous lesions, whereas ferroportin was more abundant in tuberculoid lesions. In addition, hepcidin and ferroportin were not colocalised in the biopsies from leprosy lesions. Anaemia was not commonly observed in patients with $M B$; however, the observed changes in haematologic parameters indicating altered iron metabolism appeared to result from a mixture of anaemia of inflammation and iron deficiency. Thus, iron sequestration inside host cells might play a role in leprosy by providing an optimal environment for the bacillus.

Key words: leprosy - hepcidin - iron metabolism

Iron is essential for the survival of all organisms; therefore, competition for this element is an important aspect of the host-pathogen interaction. The clinical effect of iron imbalance on infectious diseases has long been recognised: this issue was first discussed by Trousseau, in 1872, who observed that iron ingestion was associated with a poor prognosis in tuberculosis patients.

Elucidating the interaction between iron homeostasis and the immune response is crucial to improve our understanding of host-pathogen interactions in infectious diseases. Iron-binding compounds such as chelators or transporters are important targets of investigation. Hepcidin, an antimicrobial peptide produced by hepatocytes in response to excess iron or inflammation, is one of the principal iron regulatory hormones (Park et al. 2001). This iron transporter causes iron to accumulate inside cells by inducing the internalisation and destruction of ferroportin, the sole known cellular iron exporter (Delaby et al. 2005, Theurl et al. 2008). Anaemia and hypoxia decrease the production of hepcidin (Nicolas et al. 2002).

Hepcidin has been considered a link between inflammation and anaemia of chronic diseases or anaemia of inflammation (AI), which is characterised by reduced

Financial support: CNPq (401012/2005-0), Fundação Paulista contra a Hanseníase (095)

+ Corresponding author: anacarlap@gmail.com

Received 28 February 2012

Accepted 18 July 2012 levels of iron in the serum and increased iron reserves, as evidenced by elevated ferritin. Pro-inflammatory cytokines such as interleukin (IL)-6 and IL-1 connect the inflammatory response and AI by inducing hepcidin production (Nemeth et al. 2004, Lee et al. 2005). Bacterial infection of macrophages can also drive hepcidin production via Tool-like receptor (TLR)4 activation (Peyssonnaux et al. 2006).

AI seems to be an adaptive anti-infection mechanism that acts by sequestering iron within cells. However, during an infection with an intracellular pathogen, sequestering iron in macrophages can actually be detrimental to the host. In fact, the expression of ferroportin limits the growth of intracellular bacteria in macrophage cultures, whereas hepcidin expression has the opposite effect (Paradkar et al. 2008). In contrast, hepcidin inhibits microbe growth in interferon (IFN)- $\gamma$-activated macrophages infected with mycobacteria and causes direct membrane damage in $M y$ cobacterium tuberculosis (Sow et al. 2007).

The effects of iron on microbial survival are critical during therapies that modulate iron availability, as iron supplementation is associated with an increased risk of infection (reviewed by Johnson \& Wessling-Resnick 2012). Furthermore, iron chelators have been proposed as a possible therapy for chronic infections with intracellular microorganisms (Murray et al. 1978, Sazawal et al. 2006, Boelaert et al. 2007, Paradkar et al. 2008).

Leprosy is an ancient disease caused by Mycobacterium leprae, an intracellular mycobacterium that infects the skin and peripheral nerves, causing serious disabilities. Despite the efficacy of available therapies, at the beginning of 2010, there were 211,903 registered cases 
worldwide (WHO 2010). Leprosy cases present with a spectrum of clinical signs and are classified based on clinical presentation, bacillary burden and immunological and histological factors (Ridley \& Jopling 1966). Cases that fall at one end of this spectrum, referred to as the tuberculoid pole, present with few lesions and low bacillary burdens; patients at this pole develop a Thelper (Th)1 (cell-mediated) immune response against M. leprae. In contrast, patients at the lepromatous pole have a higher number of lesions and bacilli and develop a Th2 (humoral) immune response against $M$. leprae. The leprosy spectrum also contains intermediate forms of the disease referred to as borderline lepromatous (BL) or borderline tuberculoid leprosy. In addition, World Health Organization guidelines allow for the classification of leprosy cases as paucibacillary (PB) or multibacillary (MB) based on the number of lesions or the bacillary burden.

Anaemia is a common finding in patients presenting with MB leprosy (Sher et al. 1981, Lapinsky et al. 1992, Jain et al. 1995, Rea 2001). However, the exact mechanism underlying the reduction in serum iron and the effect of reduced iron levels on leprosy are not well understood. In this study, we investigated changes in parameters related to iron homeostasis in MB and considered for the first time the role of hepcidin in the progression of leprosy.

\section{SUBJECTS, MATERIALS AND METHODS}

Subjects - A total of 38 leprosy patients (LP) were selected from an outpatient unit at the Lauro de Souza Lima Institute (ILSL), São Paulo, Brazil, a tertiary leprosy reference hospital. All patients underwent clinical and histological evaluation and leprosy cases were classified based on the clinical criteria described by Ridley and Jopling (1966). The bacilloscopic index of the biopsies was calculated as suggested by Ridley (1987). Twenty-five healthy control (HC) subjects were enrolled in this study. These control subjects were employees of the ILSL and their relatives.

For the measurement of iron metabolism parameters and cytokines in the blood and hepcidin levels in the urine, we enrolled 16 patients [7 BL and 9 lepromatous lepromatous (LL)] presenting with MB leprosy undergoing multidrug therapy and 16 healthy gender and agematched control subjects. The LP were enrolled between the sixth and 12th month of treatment.

To assess hepcidin mRNA levels in the leprosy skin lesions, $13 \mathrm{MB}$ patients [2 borderline-borderline (BB), $6 \mathrm{BL}$ and $5 \mathrm{LL}$ ] were enrolled immediately after being diagnosed with leprosy and six skin biopsies without histological abnormalities were used as controls.

For immunofluorescence studies, nine skin lesion biopsies from non-treated LP representative of each leprosy pole (i.e., tuberculoid-tuberculoid and LL) were obtained from the ILSL Pathology Service; three skin biopsies with non-inflammatory alterations were included as controls.

This study was approved by the Ethical Committee of Human Experimentation at ILSL in accordance with the Helsinki Declaration of 1975. Written informed consent was obtained from all individuals included in the study.
Iron metabolism parameters and cytokine evaluations - Erythrograms were performed using an automated haematologic analyser (AVL Roche, Indianapolis, IN, USA). Serum iron levels and total iron binding capacities (TIBC) were determined by a colourimetric ferrozine-based assay (Labtest, Belo Horizonte, MG, Brazil). The transferrin saturation index (TSI) was calculated according to a standard method, as described by the assay manufacturer. The serum ferritin levels were measured by chemiluminescence (Immulite; DPC, Los Angeles, CA, USA).

The reticulocyte counts were performed after brilliant cresyl blue staining.

The serum levels of cytokines and soluble transferrin receptor (sTfR) were measured using commercial ELISA assays for IL-1 $\beta$, IL- 6 and sTfR (R\&D Systems, Minneapolis, MN, USA) and for tumour necrosis factor (TNF) (BD Biosciences, San Diego, CA, USA). The sTfR values were converted from $\mathrm{nmol} / \mathrm{L}$ to $\mathrm{mg} / \mathrm{L}$ to allow the calculation of the sTfR/log ferritin index.

Urinary hepcidin - The levels of urinary hepcidin were measured as described previously (Nemeth et al. 2004) at University of California (Los Angeles, CA, USA) by Dr Seth Rivera. In brief, cationic peptides were purified using a CM Macro-prep (Bio-Rad, Hercules, CA, USA). After purification, urinary extracts containing 0.1-0.5 mg of creatinine and a range of concentrations of a hepcidin standard were dotted on Immobilon-P membranes (Millipore Corp, Bedford, MA, USA). Hepcidin was then bound with a polyclonal rabbit antihuman hepcidin antibody produced by Dr Seth Rivera (University of California, Los Angeles) and detected by chemiluminescence using an Horseradish peroxidaseconjugated goat anti-rabbit antibody.

$R N A$ extraction and quantitative polymerase chain reaction ( $q P C R$ ) - The 5-mm punch biopsies were immersed in an RNA stabilisation solution (RNAlater; Ambion, Austin, TX, USA) and stored at $-80^{\circ} \mathrm{C}$ until processing. To collect the RNA samples, the biopsies were homogenised and the RNA was then isolated using a phenol-guanidinethiocyanate reagent (TRI Reagent; Ambion, Austin, TX, USA), chloroform and isopropanol. The total RNA ( $2 \mu \mathrm{g})$ was reverse transcribed to cDNA using $200 \mathrm{U}$ of Superscript II and $0.5 \mu \mathrm{g}$ of oligo-dT according to the manufacturer's instructions (Invitrogen, Carlsbad, CA, USA).

qPCR was performed with $1 \mu \mathrm{L}$ of cDNA in a $20-\mu \mathrm{L}$ reaction volume using iQ SYBR Green Supermix (Bio$\mathrm{Rad})$. The reactions were performed in triplicate in an iQ5 thermal cycler with a fluorescent imager (Bio-Rad). Hepcidin expression was evaluated by relative quantification and normalised to GAPDH expression as described by Pfaffl (2001). The following primer sequences were used: hepcidin forward, CCTGACCAGTGGCTCTGTTT, hepcidin reverse, CACATCCCACACTTTGATCG, GAPDH forward, TGGTATCGTGGAAGGACTC and GAPDH reverse, AGTAGAGGCAGGGATGATG.

Immunofluorescence - Fragments of tissue samples were fixed with $10 \%(\mathrm{w} / \mathrm{v})$ neutral-buffered formalin, dehydrated and stored in paraffin until analysis. Five-micron tissue sections were applied to poly-L-lysine-coated microscope slides and stored at room temperature before im- 
munofluorescence analysis. Before labelling, the sections were deparaffinised in xylene and rehydrated in a graded series of ethanol baths $(100 \%, 95 \%, 70 \%$ and $50 \%)$. Antigenic retrieval was subsequently performed with sodium citrate buffer. The slides were blocked with Protein Block Serum-Free Ready-To-Use buffer (Dako, Carpinteria, CA, USA). After being washed, the slides were incubated with the unconjugated primary anti-hepcidin (Abcam, Cambridge, UK) and anti-ferroportin antibodies (Alpha Diagnostics, San Antonio, TX, USA), washed again and incubated with the appropriate fluorochrome-conjugated (Fluorescein or Texas Red; Vector Laboratories Inc Burlingame, CA, USA) secondary antibodies as well as 4',6'diamidino-2-phenylindole. Secondary antibodies alone were used as negative controls. After being washed, the slides were mounted using $90 \%$ glycerol/phosphate buffered saline and analysed using a confocal microscope. The images were acquired using Confocal Laser Scanning Microscopy Leica TCS SPE and Leica Application Suite-Advanced Fluorescence software. Adobe Photoshop (version 4.0) was used for image processing. For quantitative analysis of hepcidin and ferroportin expression, three microscopic fields were imaged and the number of positive cells was counted in each field.

Statistical analyses - To compare the serum variables in the patients and controls, the nonparametric Mann-Whitney $U$-test was used. To compare the tuberculoid and lepromatous lesions and normal skin by immunofluorescence, the Kruskal-Wallis test was performed followed by Dunn's multiple comparison test. The correlation between the hepcidin expression level and the bacilloscopic index was calculated using a nonparametric Spearman's correlation test. The significance level was set at $\mathrm{p}<0.05$. All analyses were performed with GraphPad Instat version 3.00 for Windows (GraphPad Software, San Diego, CA, USA).

\section{RESULTS}

Iron metabolism parameters and cytokine evaluation - We first measured the haematological parameters of the MB patients and HC subjects (Fig. 1). We found significant decreases in haemoglobin $(\mathrm{Hb})$, haematocrit $(\mathrm{Ht})$, red blood cell count (RBC) and mean corpuscular volume (MCV) in the MB patients, as well as a tendency toward reduced red cell distribution width (RDW) values. However, only one patient presented critical values indicative of anaemia. The reticulocyte count was not significantly different between the LP and control subjects (Fig. 1).

Iron parameters were next evaluated in the sera of LP and controls (Fig. 1). The TIBC values were lower in LP than in healthy individuals $(\mathrm{p}=0.017)$. Moreover, we observed a marginally significant decrease in serum iron levels in LP $(p=0.054)$. The TSI values were similar between the two groups, whereas the serum ferritin levels were slightly higher in the LP. The sTfR and sTfR/log ferritin index values were significantly higher in LP than in healthy individuals $(\mathrm{p}=0.0002$ and $\mathrm{p}=$ 0.0054 , respectively).
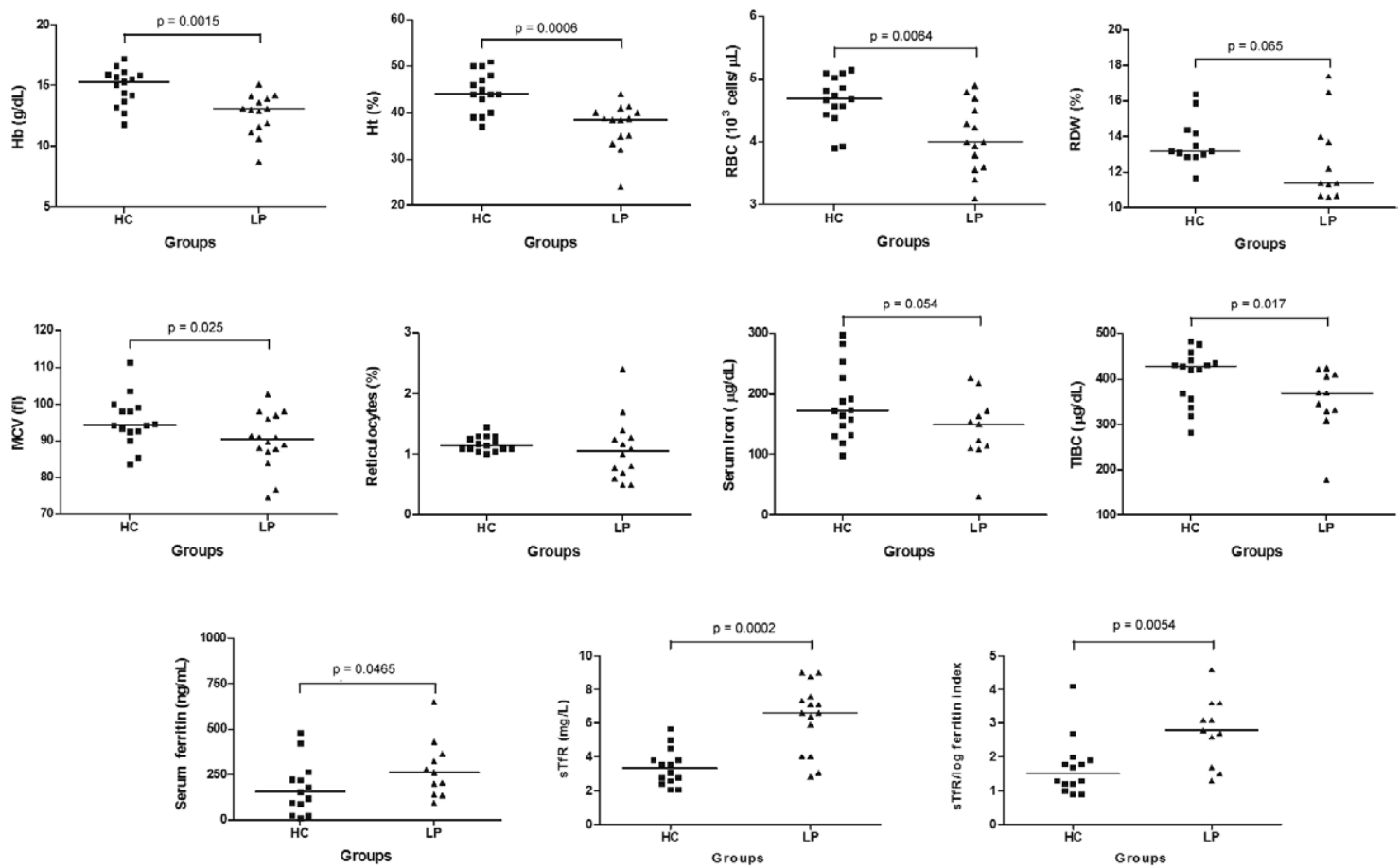

Fig. 1: measurements of haemoglobin (Hb), haematocrit (Ht), red blood cells (RBC), red cell distribution width (RDW), mean corpuscular volume (MCV), reticulocytes, serum iron, total iron binding capacity (TIBC), transferring saturation index (TSI), serum ferritin, soluble transferrin receptor (sTfR) and sTfR/log ferritin index in multibacillary leprosy patients (LP) and healthy controls (HC). Hb, Ht, RBC, MCV and TIBC showed significant decrease in LPs while serum ferritin, sTfR and sTfR/log ferritin index were increased. RDW and serum iron presented reduction with marginal significance. Bars represent medians. Nonparametric Mann-Whitney $U$-test was used to compare HCs and LPs. 
The serum levels of the pro-inflammatory cytokines IL-1 $\beta$, IL- 6 and TNF were compared in the LP and control subjects (Fig. 2). IL-1 $\beta$ levels were significantly higher in the LP than in the HC subjects $(\mathrm{p}=0.013)$; however, the levels of IL-6 and TNF were similar between the groups.

Hepcidin measurements - To better understand the role of hepcidin at sites of infection, the hepcidin levels were evaluated in the urine and skin biopsies. Urinary hepcidin levels were significantly higher in the LP than in the controls $(p=0.03)$ (Fig. 3A). In addition, the hepcidin expression levels in the biopsies of MB lesions were higher than those in the skin biopsies of healthy individuals $(\mathrm{p}=0.0022)$ (Fig. 3B).

Correlation analysis revealed that hepcidin levels are positively correlated with IL-1 $\beta$ levels in the serum of LP $(\mathrm{r}=0.563, \mathrm{p}=0.04)$ (Fig. 3C). Moreover, qPCR analysis showed a positive correlation between hepcidin expression and the bacilloscopic index in a group of $13 \mathrm{MB}$ and four PB biopsies ( $r=0.482, p=0.025)$ (Fig. 3D).

Immunofluorescence - We next evaluated the expression of hepcidin and ferroportin by immunofluorescence (Fig. 4). Ferroportin was expressed in intracellular and transmembrane compartments, whereas hepcidin was expressed more diffusely (Fig. 4). These molecules were not detected in the control samples. These results demon- strated that the expression levels of hepcidin were significantly higher in polar LP than in the control subjects ( $\mathrm{p}$ $<0.001$ ) (Fig. 5). Immunostaining of the lesions revealed the absence of colocalisation of the studied molecules in all samples. Hepcidin was more highly expressed in lepromatous lesions than in tuberculoid lesions. However, higher levels of hepcidin than ferroportin were expressed in both lepromatous lesions and tuberculoid lesions. We observed significantly weaker ferroportin expression in the control group relative to the tuberculoid group ( $\mathrm{p}<$ 0.05 ). The lepromatous samples also expressed this protein, but at a lower level than tuberculoid samples.

\section{DISCUSSION}

The manipulation of iron homeostasis by pathogens can influence the course of infectious diseases and most bacterial species possess iron acquisition mechanisms as virulence factors (Schaible \& Kaufmann 2004).

Lapinsky et al. (1992) reported the occurrence of anaemia in MB patients and classified this anaemia as AI on the basis of decreased serum iron levels and mild increases in serum ferritin concentrations.

In the present study, we have evaluated several parameters to better understand iron metabolism in LP. In particular, we have focused on the role of hepcidin in this process. Although only one leprosy patient exhibited haematological parameters consistent with anae-
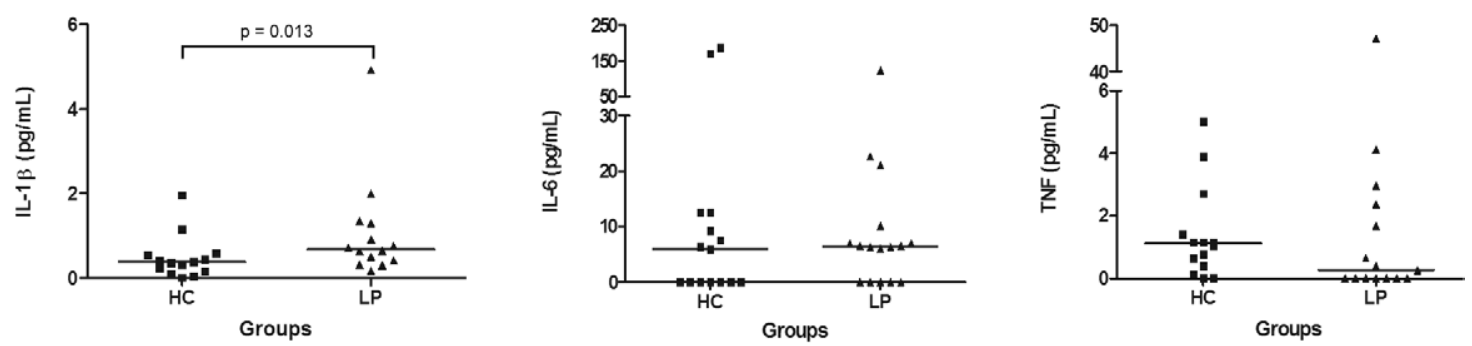

Fig. 2: measurements of interleukin (IL)-1ß, IL-6 and tumour necrosis factor (TNF) in multibacillary leprosy patients (LP) and healthy controls (HC). IL-1 $\beta$ was increased in LP. Bars represent medians. Nonparametric Mann-Whitney $U$-test was used to compare HC and LP.
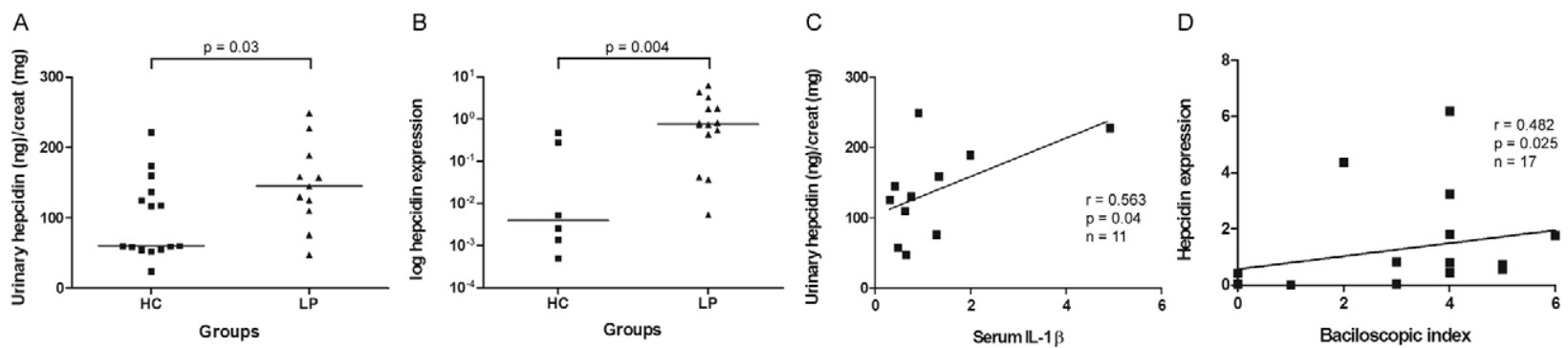

Fig. 3: evaluation of hepcidin in urine and skin samples from multibacillary leprosy patients (LP) and healthy controls (HC) and correlation of hepcidin with interleukin (IL)-1 $\beta$ and hepcidin with baciloscopic index in biopsies in LP. There is a significant increase in urinary hepcidin in the LP group. In the same way, relative expression of hepcidin mRNA is higher in biopsies from lepromatous leprosy lesions compared with normal skin. Hepcidin levels both in urine and biopsies were analyzed by nonparametric Mann-Whitney $U$-test and bars represent medians. There is a moderate correlation between urinary hepcidin and serum IL-1 $\beta$ in LP as well as a correlation between the hepcidin expression and the baciloscopic index in biopsies stained by Faraco-Fite. Nonparametric Mann-Whitney $U$-test was used to compare healthy controls and leprosy patients. Correlations were analyzed by nonparametric Spearman correlation test. 
mia, Hb, Ht, RBC, MCV and TIBC values were significantly reduced in LP. Moreover, marginally significant decreases in RDW values and serum iron levels were observed in MB patients relative to HC subjects. These findings suggest a disturbance in iron metabolism during the course of the disease. In addition, we found that LP presented increased levels of ferritin, sTfR and sTfR/ $\log$ ferritin index values. These features suggest that the LP presented a mixed anaemia including iron deficiency anaemia (IDA) and AI. Although haemolytic anaemia is a common finding after treatment with dapsone, part of the multidrug therapy for leprosy, we found no evidence of this condition when considering the direct and total bilirubin concentrations (data not shown).

The serum levels of ferritin were largely used to describe AI, since they are increased in this condition, while in IDA they are diminished. However, more recent studies have suggested that STfR and especially the STfR/log ferritin index are better parameters to distinguish AI from IDA (Punnonen et al. 1997), as the levels of sTfR are proportional to membrane expression of TfR, which increases in conditions of intracellular iron restriction. Thus, the levels of sTfR are normal in AI and elevated in IDA and combined anaemia; in contrast, the sTfR/log ferritin index values are normal in AI, strongly increased in IDA and moderately augmented in com- bined anaemia (Punnonen et al. 1997). Taken together, our results point to a complex scenario regarding iron metabolism in MB patients in which both AI and IDA are present, as we observed increased levels of serum ferritin characteristic of AI in parallel with high levels of sTfR that reflect iron deficiency. The sTfR/log ferritin index values were mildly increased in the LP relative to the $\mathrm{HC}$ subjects, providing evidence for combined anaemia during the course of MB. In this context, hepcidin certainly acts as an important mediator connecting immune response and iron metabolism in leprosy.

Through its role in regulating iron distribution, hepcidin is associated with inflammatory and infectious diseases (Armitage et al. 2011, Oustamanolakis et al. 2011). In mice, the ablation of the hepcidin gene results in severe iron overload, whereas hepcidin overexpression induces perinatal death due to iron deficiency (Nicolas et al. 2001, 2002). In our study, the hepcidin levels were higher in the urine and lesions of LP than in the urine and skin biopsies of HC subjects. Moreover, in skin biopsies, the hepcidin levels were also directly proportional to the bacilloscopic index. The induction of hepcidin by the pro-inflammatory cytokines IL-6, IL-1 and TNF is thought to result in the retention of iron inside the host cells, limiting its availability for pathogens. Pro-inflammatory cytokines are also correlated with AI in non-infectious conditions,
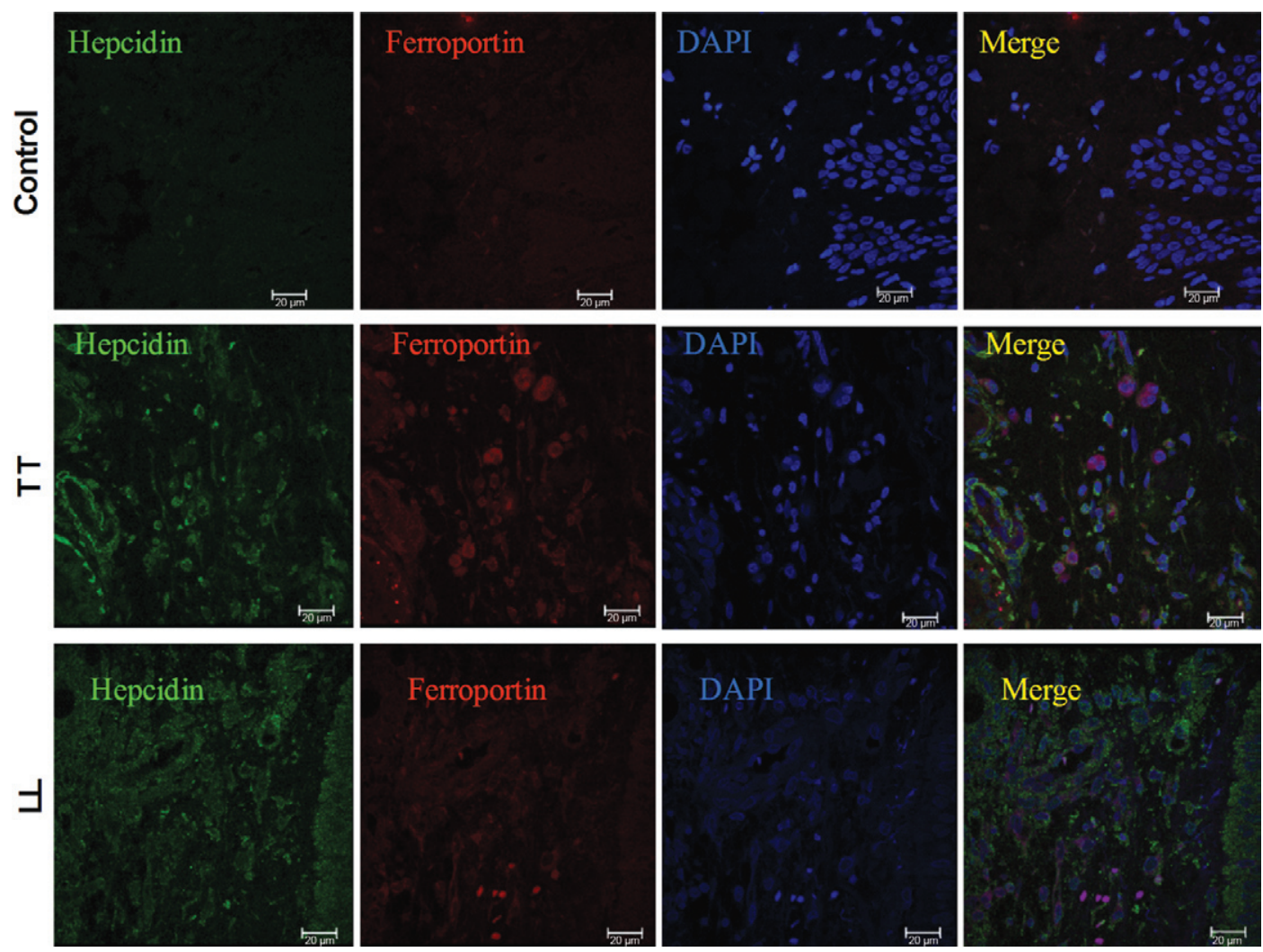

Fig. 4: expression of hepcidin and ferroportin in leprosy lesions. Biopsy samples were incubated with the appropriated fluorochrome-conjugated secondary antibodies (Texas Red or Fluorescein) as well as 4',6'-diamidino-2-phenylindole (DAPI) (blue) and analyzed by confocal microscopy. The Fig. shows the expression of hepcidin (green) and ferroportin (in red) in control, polar tuberculoid (TT) and polar lepromatous (LL) samples (original magnification: 63X). 
such as that observed in the elderly (Vanasse \& Berliner 2010). Our data showed increased IL-1 $\beta$ levels in the serum of LP and IL-1 $\beta$ levels were positively correlated with urinary hepcidin levels. In this context, the IL-1 $\beta$ production in the course of leprosy seems to favour the synthesis of hepcidin that alters iron metabolism causing a degree of AI. To our knowledge, this is the first study regarding the role of hepcidin in leprosy.

In addition to its role in promoting intracellular iron retention to restrict the growth of extracellular pathogens, hepcidin also directly acts as an antimicrobial peptide. Hepcidin partially inhibits $M$. tuberculosis growth and promotes structural damage to the bacillus (Sow et al. 2007). Despite its microbicidal role, the administration of exogenous hepcidin has been shown to enhance the growth of Chlamydia spp and Legionella pneumophila in macrophages; thus, it is possible that iron sequestration inside of the cells favours pathogen survival (Paradkar et al. 2008). In our study, hepcidin was present primarily in MB lesions, which contain a great number of bacilli. In this context, hepcidin-mediated iron sequestration could
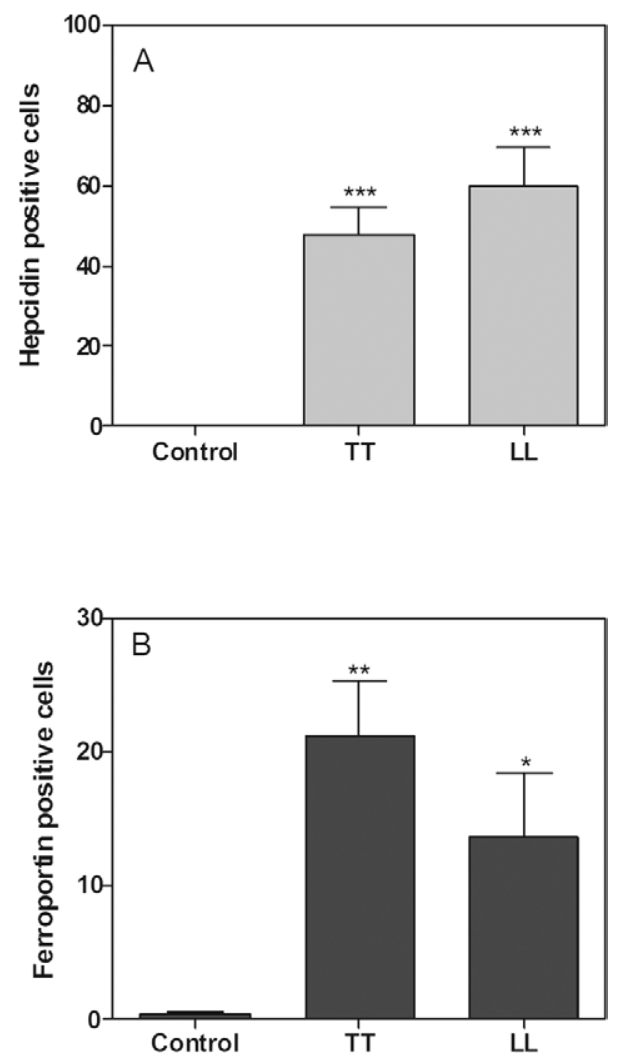

Fig. 5: quantitative analysis of the expression of hepcidin and ferroportin in leprosy samples. Results are the number of positive cells for hepcidin (A) and ferroportin (B) visualized by confocal microscopy in control, polar tuberculoid (TT) and polar lepromatous (LL) samples. The results are expressed as means \pm standard deviation for nine TT and nine LL leprosy biopsies and three normal skin biopsies. Data were analyzed by Kruskal-Wallis test followed by Dunn's Multiple Comparison Test. *: $\mathrm{p}<0.05$; **: $\mathrm{p}<0.01 ; * * * \mathrm{p}<0.001$ compared with control samples. facilitate M. leprae growth. However, in leprosy lesions, most of the bacilli have a granular appearance suggestive of bacillary death. Thus, a possible role for hepcidin in bacterial killing cannot be ruled out.

The mechanism by which hepcidin promotes intracellular iron sequestration is based on the destruction of ferroportin (Theurl et al. 2008). Ferroportin is an iron transporter that functions as a transmembrane conduit for the transfer of cellular iron to the plasma; its abolishment restricts dietary iron absorption via enterocytes and iron release by macrophages. Ferroportin mRNA levels can be elevated by erythrophagocytosis or decreased by inflammation at the individual macrophage level, whereas hepcidin is induced in response to increased iron reserves (Roy 2010). In MB biopsies, we observed many cells rich in hepcidin and fewer cells expressing ferroportin. In contrast, PB lesions exhibited fewer hepcidin-positive cells and more abundant ferroportin expression. These observations are in agreement with the model proposed for the control of iron sequestration by hepcidin and this model can help explain the optimal microenvironment provided by macrophages for M. leprae growth in susceptible individuals. Momotani et al. (1992) demonstrated that leprosy lesions express ferritin and lactoferrin, but little or no transferrin, suggesting that the granuloma is a suitable environment for iron intake by $M$. leprae. Thus, excess iron can be associated with a poor prognosis in leprosy, as observed in tuberculosis. Therefore, iron supplementation should be administered cautiously (Boelaert et al. 2007).

Several experimental animal models classically demonstrated the role of SLC11A1 (formerly natural resistance-associated macrophage protein 1), a divalent metal transporter, in mice resistance to intracellular pathogens such as Mycobacterium, Salmonella and Leishmania (Vidal et al. 1995, Canonne-Hergaux et al. 1999). In humans, SLC11A1 is located at the phagosomal membrane and influences the iron content and pathogen survival in macrophages. Genetic associations between this gene and leprosy provide more evidence of the importance of iron transport proteins to its pathogenesis (Abel et al. 1998, Meisner et al. 2001). In the present study, the focus on different iron transporters complements and extends the results previously described. This study constitutes an extensive investigation exploring the interplay between multiple iron transporters and intracellular microorganisms inside the macrophage.

Taken together, our results demonstrate for the first time increased levels of hepcidin in the urine and lesions of MB patients presenting a combination of AI and IDA. The elevated hepcidin levels in LP could favour iron retention inside macrophages and thereby support $M$. leprae growth. Further studies are necessary to elucidate the exact influence of hepcidin on M. leprae biology and leprosy pathophysiology.

\section{ACKNOWLEDGEMENTS}

To Dr Seth Rivera, for performing hepcidin measurements and for his valuable suggestions, to Neusa Broch, from the Rondonópolis Health Service, and to the staff from the Clinical Laboratory of the ILSL, for technical assistance. 


\section{REFERENCES}

Abel L, Sánchez FO, Oberti J, Thuc NV, Hoa LV, Lap VD, Skamene E, Lagrange PH, Schurr E 1998. Susceptibility to leprosy is linked to the human NRAMP1 gene. J Infect Dis 177: 133-145.

Armitage AE, Eddowes LA, Gileadi U, Cole S, Spottiswoode N, Selvakumar TA, Ho LP, Townsend AR, Drakesmith H 2011. Hepcidin regulation by innate immune and infectious stimuli. Blood 118: 4129-4139.

Boelaert JR, Vandecasteele SJ, Appelberg R, Gordeuk VR 2007. The effect of the host's iron status on tuberculosis. J Infect Dis 195: $1745-1753$

Canonne-Hergaux F, Gruenheid S, Govoni G, Gros P 1999. The Nrampl protein and its role in resistance to infection and macrophage function. Proc Assoc Am Physicians 111: 283-289.

Delaby C, Pilard N, Gonçalves AS, Beaumont C, Canonne-Hergaux F 2005. Presence of the iron exporter ferroportin at the plasma membrane of macrophages is enhanced by iron loading and down-regulated by hepcidin. Blood 106: 3979-3984.

Jain A, Mukherjee A, Chattopadhya D, Saha K 1995. Biometals in skin and sera of leprosy patients and their correlation to trace element contents of $M$. leprae and histological types of the disease - a comparative study with cutaneous tuberculosis. Int $J$ Lepr Other Mycobact Dis 63: 249-258.

Johnson EE, Wessling-Resnick M 2012. Iron metabolism and the innate immune response to infection. Microbes Infect 14: 207-216.

Lapinsky SE, Baynes RD, Schulz EJ, MacPhail AP, Mendelow B, Lewis D, Bothwell TH 1992. Anaemia, iron-related measurements and erythropoietin levels in untreated patients with active leprosy. J Intern Med 232: 273-278.

Lee P, Peng H, Gelbart T, Wang L, Beutler E 2005. Regulation of hepcidin transcription by interleukin-1 and interleukin-6. Proc Natl Acad Sci USA 102: 1906-1910.

Meisner SJ, Mucklow S, Warner G, Sow SO, Lienhardt C, Hill AV 2001. Association of NRAMP1 polymorphism with leprosy type but not susceptibility to leprosy per se in west Africans. Am J Trop Med Hyg 65: 733-735.

Momotani E, Wuscher N, Ravisse P, Rastogi N 1992. Immunohistochemical identification of ferritin, lactoferrin and transferrin in leprosy lesions of human skin biopsies. J Comp Pathol 106: 213-220.

Murray MJ, Murray AB, Murray MB, Murray CJ 1978. The adverse effect of iron repletion on the course of certain infections. $\mathrm{Br} \mathrm{Med}$ $J$ 2: 1113-1115.

Nemeth E, Rivera S, Gabayan V, Keller C, Taudorf S, Pedersen BK, Ganz T 2004. IL-6 mediates hypoferremia of inflammation by inducing the synthesis of the iron regulatory hormone hepcidin. J Clin Invest 113: 1271-1276.

Nicolas G, Bennoun M, Devaux I, Beaumont C, Grandchamp B, Kahn A, Vaulont S 2001. Lack of hepcidin gene expression and severe tissue iron overload in upstream stimulatory factor 2 (USF2) knockout mice. Proc Natl Acad Sci USA 98: 8780-8785.

Nicolas G, Bennoun M, Porteu A, Mativet S, Beaumont C, Grandchamp B, Sirito M, Sawadogo M, Kahn A, Vaulont S 2002. Severe iron deficiency anemia in transgenic mice expressing liver hepcidin. Proc Natl Acad Sci USA 99: 4596-4601.
Oustamanolakis P, Koutroubakis IE, Messaritakis I, Malliaraki N, Sfiridaki A, Kouroumalis EA 2011. Serum hepcidin and prohepcidin concentrations in inflammatory bowel disease. Eur J Gastroenterol Hepatol 23: 262-268.

Paradkar PN, De Domenico I, Durchfort N, Zohn I, Kaplan J, Ward DM 2008. Iron depletion limits intracellular bacterial growth in macrophages. Blood 112: 866-874.

Park CH, Valore EV, Waring AJ, Ganz T 2001. Hepcidin, a urinary antimicrobial peptide synthesized in the liver. J Biol Chem 276: 7806-7810.

Peyssonnaux C, Zinkernagel AS, Datta V, Lauth X, Johnson RS, Nizet V 2006. TLR4-dependent hepcidin expression by myeloid cells in response to bacterial pathogens. Blood 107: 3727-3732.

Pfaffl MW 2001. A new mathematical model for relative quantification in real-time RT-PCR. Nucleic Acids Res 29: 2002-2007.

Punnonen K, Irjala K, Rajamäki 1997. A serum transferrin receptor and its ratio to serum ferritin in the diagnosis of iron deficiency. Blood 89: 1052-1057.

Rea TH 2001. Decreases in mean hemoglobin and serum albumin values in erythema nodosum leprosum and lepromatous leprosy. Int J Lepr Other Mycobact Dis 69: 318-327.

Ridley DS 1987. Skin biopsy in leprosy, 2nd ed., Ciba-Geigy, Basel, $63 \mathrm{pp}$.

Ridley DS, Jopling WH 1966. Classification of leprosy according to immunity. A five-group system. Int J Lepr Other Mycobact Dis 34: $255-273$.

Roy CN 2010. Anemia of inflammation. Hematology Am Soc Hematol Educ Program 2010: 276-280.

Sazawal S, Black RE, Ramsan M, Chwaya HM, Stoltzfus RJ, Dutta A, Dhingra U, Kabole I, Deb S, Othman MK, Kabole FM 2006. Effects of routine prophylactic supplementation with iron and folic acid on admission to hospital and mortality in preschool children in a high malaria transmission setting: community-based, randomised, placebo-controlled trial. Lancet 367: 133-143.

Schaible UE, Kaufmann SH 2004. Iron and microbial infection. Nat Rev Microbiol 2: 946-953.

Sher R, Shulman G, Baily P, Politzer WM 1981. Serum trace elements and vitamin A in leprosy subtypes. Am J Clin Nutr 34: 1918-1924.

Sow FB, Florence WC, Satoskar AR, Schlesinger LS, Zwilling BS, Lafuse WP 2007. Expression and localization of hepcidin in macrophages: a role in host defence against tuberculosis. $J$ Leukoc Biol 82: 934-945.

Theurl I, Theurl M, Seifert M, Mair S, Nairz M, Rumpold H, Zoller H, Bellmann-Weiler R, Niederegger H, Talasz H, Weiss G 2008. Autocrine formation of hepcidin induces iron retention in human monocytes. Blood 111: 2392-2399.

Trousseau A 1872. True and false chlorosis. Lectures on Clinical Medicine 5: 95-117.

Vanasse GJ, Berliner N 2010. Anemia in elderly patients: an emerging problem for the 21st century. Hematology Am Soc Hematol Educ Program 2010: 271-275.

Vidal S, Gros P, Skamene E 1995. Natural resistance to infection with intracellular parasites: molecular genetics identifies Nramp1 as the Bcg/Ity/Lsh locus. J Leukoc Biol 58: 382-390.

WHO - World Health Organization 2010. Global leprosy situation. Wkly Epidemiol Rec 85: 337-348. 